\title{
Agrobiodiversity of Home Gardens in Selected Marginal Upland Villages of Inopacan, Leyte, Philippines
}

\author{
Beatriz S. Belonias, Czarina S. Platino and Jessa T. Malanguis \\ Department of Biological Sciences, College of Arts and Sciences \\ Visayas State University, Visca, 6521-A Baybay City, Philippines
}

\section{ABSTRACT}

Home gardens are subsistence crop production systems that significantly contribute to the socio-ecological resilience of peasant communities reducing vulnerability and ensuring food security. Because of their small size, they are neglected repositories of biological diversity. This paper describes the agrobiodiversity and management of home gardens in about 79 households in three marginal upland villages of Inopacan, Leyte. Semi-structured interviews and actual home garden visits were done to collect data. Results show that home gardens studied had high species diversity and exhibited a multilayered structure. A total of 171 species in 74 families and 146 genera were documented. The composites (Asteraceae) had the most number of genera and species followed by the orchids (Orchidaceae), legumes (Fabaceae) and aroids (Araceae). More than $70 \%$ of the species were exotics which were mostly ornamental plants. Erect herbs were the most common, followed by trees, shrubs, herbaceous vines and epiphytes. Majority of the species were ornamentals followed by fruit trees, medicinal plants, vegetables and spice plants, grown primarily for home use (62\%)rather than as source of income. Established and managed by family members who provide free labor, the gardens mostly utilized cost-free planting materials and with no fertilizer or pesticide application.

Key words: agroecosystem, biodiversity, subsistence farming, backyard garden, medicinal plants

Correspondence: B.S. Belonias Address: Graduate School, Visayas State University, Visca, Baybay City, Leyte, 6521A Philippines Email: bingsat_b62@yahoo.com

DOI: $10.32945 /$ atr36s4.2014 
Agrobiodiversity of home gardens in marginal upland Villages of Inopacan

\section{INTRODUCTION}

In the humid tropics, home gardens are a common feature in village communities. Also called backyard gardens, these are small areas planted to various plant species situated in the immediate vicinity of human dwellings (Sunwar, 2006). These are often separated from the surrounding landscape by fences, hedges, trees or other barriers (Guarino and Hoogendijk, 2004). Although considered as a subsistence type of agriculture due to their small size and volume of production, they are multifunctional agroecosystems (Galluzi et al., 2010). Home gardens significantly contribute to the socio-ecological resilience of peasant communities reducing vulnerability and ensuring food security (Buchman, 2009). Throughout history, family members strengthen family ties as they work together in home gardens. They grow food, spice, ornamental and medicinal plants, fruit trees and other species, usually intended for home use but can sometimes be a source of additional income for the family. In some communities, home gardens play a role in cultural festivities and religious rites (Thrinh et al, 2003). In many countries around the world, home gardens have become part and parcel of life in the countryside.

Traditional home gardens are typically characterized by having a complex multilayered architecture and high species diversity (Soemarwoto, 1987, Coomes and Ban (2004)), where a variety of herbs, shrubs, epiphytes, vines and trees are grown together. Smith et al. (2006) explained that such stratified and highly diverse structure makes home gardens a generally sustainable and resilient ecosystem. Their close proximity to human dwellings ensures a more personalized type of management, making the biotic and abiotic conditions in the home garden unique or considerably different from the surrounding environment (Guarino and Hoogendijk, 2004).

In recent years, the threat of biodiversity loss both in natural environments and agricultural systems has received increasing attention. As a micro-agroecosystem of diverse structure, home gardens are important repositories of plant genetic diversity and thus could function effectively for the in situ conservation of plant genetic resources. The study of Eyzaguirre and Watson (2001) pointed out that home gardens are crucial reservoirs of inter- and intra-genetic crop genetic diversity. Galuzzi et al. (2010) stressed that a significant portion of the world's agrobiodiversity can be found in complex agroecosystems managed by small farmers. In situ conservation of crop species and landraces in these 
complex ecosystems ensures their continuous adaptation to climate change.

The most important contribution of home gardening for conservation purposes resides in its capacity to represent agrobiodiversity at multiple levels over small spaces (Hodgkin 2001). Furthermore, they are often utilized as preliminary venues for new crops, as nurseries for growing plants which will be transferred for planting in open fields and domestication sites of weedy forms (Kulpa and Hanelt 1981; Leiva et al. 2001), which may also be used directly within the household.

According to Trinh et al. (2003) home gardening is one of the most under-researched topics. In a review done by Galuzzi et al. (2010), they found that much of past research on home gardens has been done in developed countries and focused more on their role as a distinct ecological or cultural entity. Little attention has been given to their role as critical repositories of plant genetic diversity. This study was therefore done to assess the agrobiodiversity of home gardens in selected sites in Eastern Visayas with emphasis on those found in marginal degraded uplands considering that these areas are highly vulnerable to climate change. Specifically, it aimed to identify the species and varieties of plants raised in the gardens, document their economic uses and, characterize the structure and management of these home gardens.

\section{MATERIALS AND METHODS}

\section{The Study Site}

The town of Inopacan lies on the southwestern portion of the province of Leyte, Philippines, some $130 \mathrm{~km}$ from the capital city of Tacloban. It is a fourth class municipality and has a total land area of $94.62 \mathrm{~km}^{2}$. It is politically divided into 20 barangays and as of 2010 , the population was about 19,904 people (www.inopacan.gov.ph).

A reconnaissance survey was first conducted in the upland villages of Inopacan to select suitable marginal upland sites for the study. Three villages were selected, namely; Brgy. Linao, Brgy. Guinsangaan and Brgy. Tao-taon (Fig. 1) These are three adjacent villages or barangays situated 
towards the southeastern part of the town. The interior upland portions of these villages have wide stretches of hilly open grasslands where the soil is poor and infertile. Asio, et al (2014) described the soil in the marginal uplands of Inopacan as highly acidic and low in organic matter, total nitrogen and available P. Such soil characteristics aggravated by high temperatures and water scarcity make these sites unsuitable for crop production.

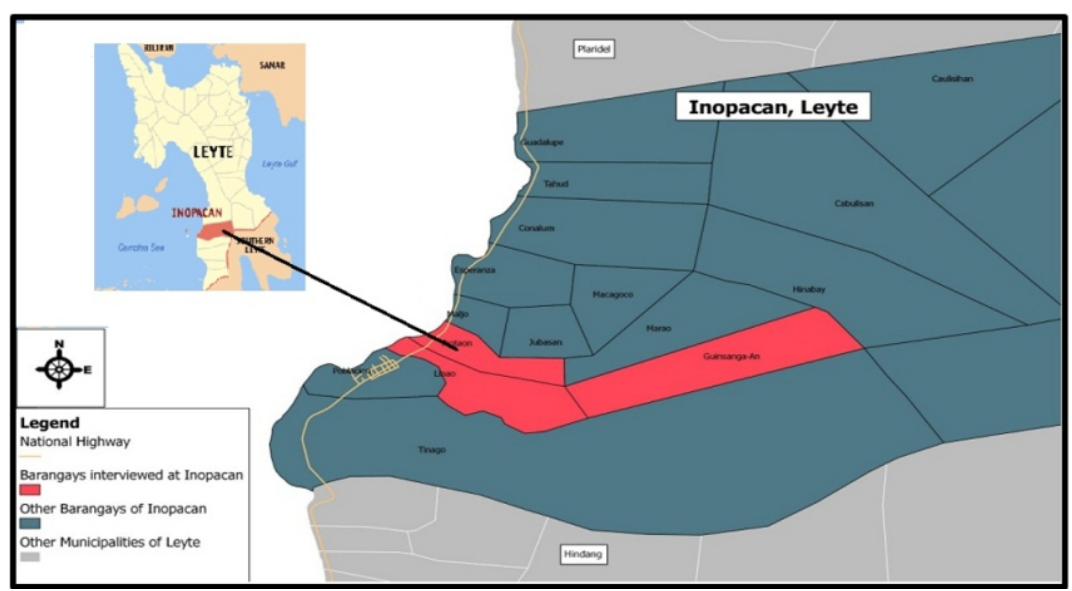

Figure 1. Map of Leyte province and Inopacan town showing the location of the study sites

\section{Data Collection}

A total of 79 respondents having home gardens were interviewed right in their homes using a survey questionnaire. Of this, 11 were from Linao, 23 from Guinsangaan and 45 from Tao-taon, representing 14\%, 29\%, and $57 \%$ of the total number of respondents, respectively. These respondents were randomly picked from an official list of residents living in the marginal upland areas of these villages. Actual observations of their home gardens were also done to identify the species and varieties grown, collect sample specimens and take photos for documentation. Voucher specimens of all species documented in the survey and home visits were processed into herbarium specimens and deposited in the VSU Herbarium, Visayas State University, Visca, Baybay City, Leyte. 


\section{Specimen Identification}

All plant species recorded in the surveys and home garden/farm visits were identified and classified using every taxonomic reference available but Co's Digital Flora (Pielser et al, 2012-onwards) and the revisions of the Flora Malesiana series ) Steenis, (1950 onwards) were heavily relied on.

\section{RESULTS AND DISCUSSION}

\section{Diversity and Structure of Home Gardens}

The home gardens studied were found to exhibit high species richness and diversity. A total of 171 species distributed in 74 families and 146 genera were documented. (Table 1). The family Asteraceae/Compositae was the most represented in terms of number of genera (9) and species (10) followed by Orchidaceae $(7,8)$, Fabaceae $(7,7)$, Araceae $(7,7)$, Zingiberaceae $(6,7)$, Lamiaceae $(5,6)$, Poaceae $(4,5)$, Cucurbitaceae $(4,4)$, Asparagaceae $(4,4)$, Apocynaceae $(4,4)$ and Meliaceae $(4,4)$. One hundred twenty-one (12) species or more than $70 \%$ of the total number of species documented are introduced or exotic species, especially for ornamental plants. It appears therefore that non-native species comprised a considerable part in the composition of home gardens in the marginal uplands studied.

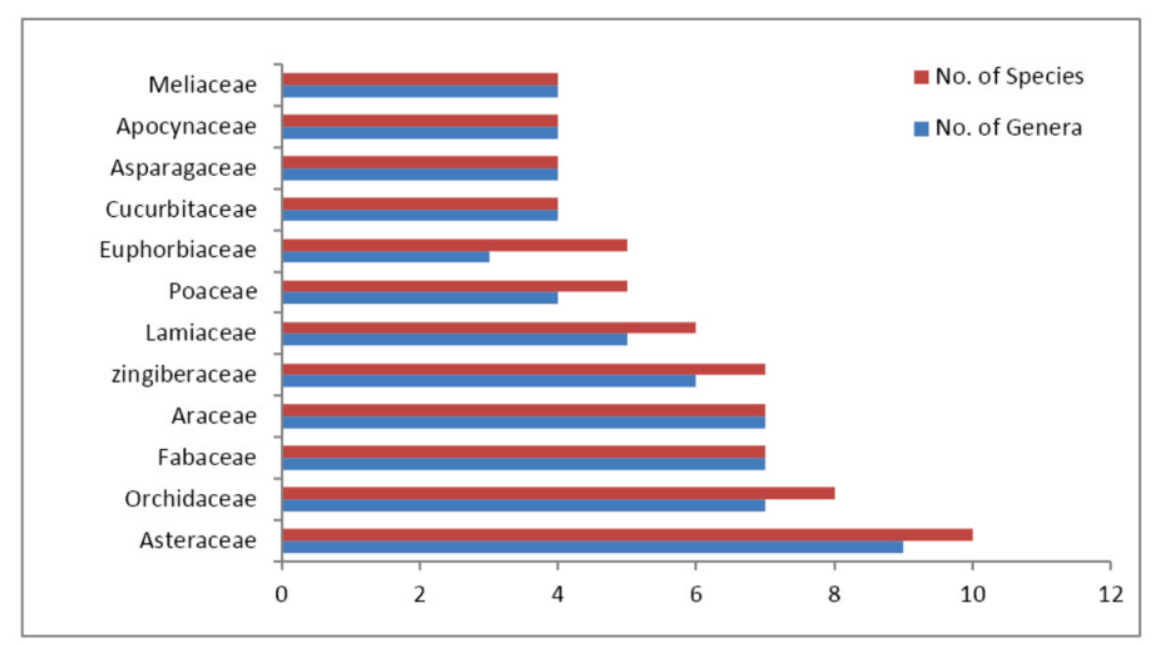

Figure 2. Plant families having the most number of genera and species 
Table 1. Plant species recorded in the home gardens of the marginal upland villages of Inopacan, Leyte.

\begin{tabular}{|c|c|c|c|c|}
\hline Family & Scientific Name & Local Name & Economic Uses & Origin \\
\hline \multirow[t]{2}{*}{ Acanthaceae } & $\begin{array}{l}\text { Justicia brandegeeana Wassh.\& } \\
\text { L.B.Sm. }\end{array}$ & Shrimp Plant (Engl.) & Ornamental & Introduced \\
\hline & $\begin{array}{l}\text { Pseuderanthemum reticulatum } \\
\text { (Hook.f.) Radlk. }\end{array}$ & Pasaw & Ornamental & \multirow[b]{2}{*}{ Introduced } \\
\hline Adiantaceae & Adiantum L. & Lamon-lamon & Medicinal & \\
\hline Agavaceae & Cordyline fruticosa (L.) A.Chev. & Baston, Ti plant & Ornamental & Native \\
\hline \multirow[t]{2}{*}{ Amaranthaceae } & $\begin{array}{l}\text { Alternanthera ficoidea (L.) P. } \\
\text { Beauv. }\end{array}$ & Kutcharita & Ornamental & \multirow{2}{*}{$\begin{array}{l}\text { Introduced } \\
\text { Introduced }\end{array}$} \\
\hline & Celosia cristata L. & Pasong-pasong & Ornamental & \\
\hline \multirow[t]{4}{*}{ Amaryllidaceae } & Allium fistulosum $\mathrm{L}$. & Sibuyas- dahon & spice, medicinal & Introduced \\
\hline & Allium sp. L. & Sibojing & Spice & Introduced \\
\hline & Allium sp. L. & Ganda & Spice & Introduced \\
\hline & Crinum latifolium $\mathrm{L}$. & Lirio & Ornamental & Native \\
\hline \multirow[t]{3}{*}{ Anacardiaceae } & Mangifera indica $\mathrm{L}$. & Manga & Fruit & Introduced \\
\hline & Anacardium occidentale & Kasoy & Fruit & Introduced \\
\hline & Spondias pinnata (L. f.) Kurz. & Libas & Fruit & Native \\
\hline Annonaceae & Annona muricata $\mathrm{L}$. & Abana, Guyabano & Fruit, medicinal & Introduced \\
\hline \multirow[t]{4}{*}{ Apocynaceae } & Allamanda cathartica L. & Yellow Bell & Ornamental & Introduced \\
\hline & Catharanthus roseus (L.) G.Don & Kumintang & Ornamental & Introduced \\
\hline & Plumeria rubra L. & Calachuching Pula & Ornamental & Introduced \\
\hline & $\begin{array}{l}\text { Tabernaemontana pandacaqui } \\
\text { Lam. }\end{array}$ & Pandakaki & Ornamental & Native \\
\hline \multirow[t]{7}{*}{ Araceae } & Aglaonema commutatum Schott & Aglaonema & Ornamental & \multirow{2}{*}{$\begin{array}{l}\text { Native } \\
\text { Introduced }\end{array}$} \\
\hline & Anthurium aucbeanum & Anthurium & Ornamental & \\
\hline & Caladium bicolor (Aiton) Vent. & Gabi-gabi & Ornamental & Introduced \\
\hline & Colocasia esculenta (L.) Schott & Gabi & $\begin{array}{l}\text { Root crop, } \\
\text { vegetable }\end{array}$ & Introduced \\
\hline & $\begin{array}{l}\text { Cyrtosperma merkusii (Hassk.) } \\
\text { Schott }\end{array}$ & Palau & Root crop & Introduced \\
\hline & $\begin{array}{l}\text { Dieffenbachia picta (Lodd.) } \\
\text { Schott. }\end{array}$ & $\begin{array}{l}\text { Dieffenbachia } \\
\text { (Engl.) }\end{array}$ & Ornamental & Introduced \\
\hline & $\begin{array}{l}\text { Spathiphyllum commutatum } \\
\text { Schott }\end{array}$ & Peace Lily & Ornamental & Native \\
\hline \multirow[t]{2}{*}{ Araliaceae } & Polyscias fruticosa (L.) Harms & Kalipay & Ornamental & Introduced \\
\hline & $\begin{array}{l}\text { Schefflera arboricola (Hayata) } \\
\text { Kanehira }\end{array}$ & Five fingers & Ornamental & Introduced \\
\hline Araucariaceae & $\begin{array}{l}\text { Araucaria heterophylla (Salisb.) } \\
\text { Franco }\end{array}$ & Pine tree & Ornamental & Introduced \\
\hline \multirow[t]{3}{*}{ Arecaceae } & Livistona rotundifolia (Lam) Mart & Anahaw & Ornamental & Native \\
\hline & Veitchia merrillii (Becc.) Bec. & Lubi-lubi & Ornamental & Native \\
\hline & Cocos nucifera $\mathrm{L}$. & Lubi & $\begin{array}{l}\text { food, lumber, } \\
\text { firewood, } \\
\text { medicinal }\end{array}$ & Native \\
\hline
\end{tabular}


Table1. Continuation.

\begin{tabular}{|c|c|c|c|c|}
\hline Family & Scientific Name & Local Name & Economic Uses & Origin \\
\hline \multirow[t]{4}{*}{ Asparagaceae } & Asparagus sprengeri Regel & Asparagus & Ornamental & Introduced \\
\hline & Dracaena fragrans (L.) Ker Gawl. & Fortune plant & Ornamental & Introduced \\
\hline & Sansevieria trifasciata Prain & Espada-espada & Ornamental & Introduced \\
\hline & $\begin{array}{l}\text { Chlorophytum comosum } \\
\text { (Thunb.) Jacques }\end{array}$ & Ribbon plant & Ornamental & Introduced \\
\hline Aspleniaceae & Asplenium nidus $\mathrm{L}$. & Mana-o & Ornamental & Native \\
\hline \multirow[t]{10}{*}{ Asteraceae } & Artemisia vulgaris $\mathrm{L}$. & Hilbas & Medicinal & Native \\
\hline & Aster ericoides L. & Baby's Breath & Ornamental & Introduced \\
\hline & Blumea balsamifera (L.) DC & Gabon & Medicinal & Native \\
\hline & $\begin{array}{l}\text { Dendranthema indica (L.) Des } \\
\text { Moul. }\end{array}$ & Chrysanthemum & Ornamental & Native \\
\hline & Cosmos bipinnatus Cav. & Cosmos & Ornamental & Introduced \\
\hline & Helianthus annuus $\mathrm{L}$. & Sunflower & Ornamental & Introduced \\
\hline & Cosmos caudatus Kunth & Cosmos & Ornamental & Introduced \\
\hline & Tagetes erecta $\mathrm{L}$. & Marigold & Ornamental & Introduced \\
\hline & $\begin{array}{l}\text { Pseudoelephantopus spicatus (B. } \\
\text { Juss.) Gleason }\end{array}$ & Kuko's Banog & Medicinal & Introduced \\
\hline & $\begin{array}{l}\text { Gerbera jamesoides Bolus ex } \\
\text { Hooker } \mathrm{f} \text {. }\end{array}$ & Daisy & Ornamental & Introduced \\
\hline Balsaminaceae & Impatiens balsamina $\mathrm{L}$. & Swangga & Ornamental & Introduced \\
\hline Basellaceae & Basella alba L. & Alugbati & Vegetable & Native \\
\hline Begoniaceae & Begonia L. & Begonia & Ornamental & Native \\
\hline Bixaceae & Bixa orellana L. & Achuete & Spice/condiment & Introduced \\
\hline Bombacaceae & Durio zibethinus Murray & Durian & Fruit & Native \\
\hline Brassicaceae & Brassica rapa $\mathrm{L}$. & Pechay & Vegetable & Introduced \\
\hline \multirow[t]{2}{*}{ Bromeliaceae } & Ananas comosus (L.) Merr. & Pinya & Fruit & Introduced \\
\hline & Guzmania lingulata $\mathrm{Mez}$ & Scarlet & Ornamental & Introduced \\
\hline Burseraceae & Canarium ovatum Engl & Pili & fruit, lumber & Native \\
\hline Cactaceae & Cactus sp. & Cactus & Ornamental & Introduced \\
\hline Caricaceae & Carica papaya $\mathrm{L}$. & Kapayas, Papaya & Fruit & Introduced \\
\hline Clusiaceae & Garcinia mangostana L. & Mangosteen & Fruit, medicinal & Introduced \\
\hline Commelinaceae & Tradescantia spathacea Sw. & $\begin{array}{l}\text { Bangka-bangkaan, } \\
\text { Rhoeo }\end{array}$ & Ornamental & Introduced \\
\hline \multirow[t]{2}{*}{ Convolvulaceae } & Ipomoea batatas (L.) Lam. & Camote & Root crop & Introduced \\
\hline & Ipomoea aquatica Forssk. & $\begin{array}{l}\text { Tangkong, } \\
\text { Kangkong }\end{array}$ & Vegetable & Introduced \\
\hline Crassulaceae & Kalanchoe pinnata (Lam.) Pers. & Hanlilika & Medicinal & Introduced \\
\hline \multirow[t]{4}{*}{ Cucurbitaceae } & Cucurbita maxima Duchesne & Kalabasa & Vegetable & Introduced \\
\hline & $\begin{array}{l}\text { Lagenaria siceraria Molina } \\
\text { Standl. }\end{array}$ & Upo, Balantiyong & Vegetable & Introduced \\
\hline & Luffa acutangula (L.) Roxb. & Sikwa & Vegetable & Introduced \\
\hline & Momordica charantia L. & Paliya, Ampalaya & $\begin{array}{l}\text { medicinal, } \\
\text { vegetable }\end{array}$ & Introduced \\
\hline Cupressaceae & Thuja orientalis L. & Cypress & Ornamental & Introduced \\
\hline
\end{tabular}


Table1. Continuation.

\begin{tabular}{|c|c|c|c|c|}
\hline Family & Scientific Name & Local Name & Economic Uses & Origin \\
\hline Cycadaceae & Cycas revoluta Thunb. & Pitogo & Ornamental & Introduced \\
\hline \multirow[t]{2}{*}{ Dioscoreaceae } & Dioscorea alata L. & Ube & Root crop & Native \\
\hline & Dioscorea bulbifera L. & Ube & Root crop & Native \\
\hline Ebenaceae & Diospyros philippinensis A DC & Mabolo, Kamagong & fruit, timber & \multirow{2}{*}{$\begin{array}{l}\text { Native } \\
\text { Introduced }\end{array}$} \\
\hline Equisetaceae & Equisetum sp. L. & Horsetail & Ornamental & \\
\hline \multirow[t]{6}{*}{ Euphorbiaceae } & Codiaeum variegatum (L.)A. Juss & San Francisco & Ornamental & Introduced \\
\hline & $\begin{array}{l}\text { Euphorbia pulcherrima Willd. ex } \\
\text { Klotchzsch }\end{array}$ & Poinsettia & Ornamental & Introduced \\
\hline & Euphorbia neriifolia L. & Suro-suro & Medicinal & Introduced \\
\hline & Jatropha curcas L. & Tuba-tuba & Medicinal & Introduced \\
\hline & Jatropha gossypiifolia L. & Tuba-tubang Tapol & & Introduced \\
\hline & Jatropha podagrica Hook. & Ginseng & Medicinal & Introduced \\
\hline \multirow[t]{7}{*}{ Fabaceae } & $\begin{array}{l}\text { Gliricidia sepium } \\
\text { (Jacq.) Kunth ex Walp. }\end{array}$ & $\begin{array}{l}\text { Madre de cacao, } \\
\text { Kakawate }\end{array}$ & firewood, fencing & Introduced \\
\hline & $\begin{array}{l}\text { Leucaena leucocephala (Lam.) De } \\
\text { Wit }\end{array}$ & Ipil-ipil & firewood, fencing & Introduced \\
\hline & $\begin{array}{l}\text { Psophocarpus tetragonolobus (L.) } \\
\text { D.C }\end{array}$ & $\begin{array}{l}\text { Garbanzos, } \\
\text { Kalabantos } \\
\text { Kalabantos }\end{array}$ & Vegetable & Introduced \\
\hline & Pterocarpus indicus Willd. & Narra, Naga & Timber & Native \\
\hline & Tamarindus indica $\mathrm{L}$. & Sambag, Sampalok & $\begin{array}{l}\text { fruit, } \\
\text { spice/condiment }\end{array}$ & Introduced \\
\hline & Vigna unguiculata (L.) Walp. & Batong & Vegetable & Introduced \\
\hline & $\begin{array}{l}\text { Flemingia macrophylla (Willd.) } \\
\text { Merr., }\end{array}$ & Malabalatong & Hedge plant & Native \\
\hline Gnetaceae & Gnetum gnemon L. & Bago & Vegetable & Native \\
\hline Heliconiaceae & Heliconia psittacorum L.f & Saging-saging & Ornamental & Introduced \\
\hline Hydrangeaceae & $\begin{array}{l}\text { Hydrangea macrophylla (Thunb.) } \\
\text { Ser }\end{array}$ & Millions & Ornamental & Introduced \\
\hline \multirow[t]{6}{*}{ Lamiaceae } & $\begin{array}{l}\text { Orthosiphon aristatus } \\
\text { (Blume) Miq. }\end{array}$ & Balbas- pusa & Medicinal & Native \\
\hline & $\begin{array}{l}\text { Plectranthus amboinicus (Lour.) } \\
\text { Spreng }\end{array}$ & Karabo & spice, medicinal & Introduced \\
\hline & $\begin{array}{l}\text { Plectranthus scutellarioides (L.) } \\
\text { R.Br.. }\end{array}$ & Mayana & $\begin{array}{l}\text { ornamental, } \\
\text { medicinal }\end{array}$ & Native \\
\hline & Hyptis suaveolens (L.) Poit & & Medicinal & Introduced \\
\hline & Mentha arvensis $\mathrm{L}$. & Mentol & Medicinal & Introduced \\
\hline & Mentha cordifolia Opiz & Yerba Buena & Medicinal & Introduced \\
\hline Lauraceae & Persea americana Mill. & Avocado & Fruit, medicinal & Introduced \\
\hline Lythraceae & Lagerstroemia speciosa (L.)Pers. & Banaba & Medicinal & \multirow{2}{*}{$\begin{array}{l}\text { Native } \\
\text { Introduced }\end{array}$} \\
\hline \multirow[t]{3}{*}{ Malvaceae } & $\begin{array}{l}\text { Abelmoschus esculentus } \\
\text { (L.) Moench }\end{array}$ & Okra & Vegetable & \\
\hline & Hibiscus rosa-sinensis $\mathrm{L}$. & $\begin{array}{l}\text { Antuwanga, } \\
\text { Gumamela }\end{array}$ & $\begin{array}{l}\text { Ornamental, } \\
\text { medicinal }\end{array}$ & \multirow{2}{*}{$\begin{array}{l}\text { Introduced } \\
\text { Introduced }\end{array}$} \\
\hline & Gossypium hirsutum & Gapas & Cotton fiber & \\
\hline
\end{tabular}


Table1. Continuation.

\begin{tabular}{|c|c|c|c|c|}
\hline Family & Scientific Name & Local Name & Economic Uses & Origin \\
\hline \multirow[t]{4}{*}{ Meliaceae } & $\begin{array}{l}\text { Lansium domesticum } \\
\text { (Osbeck) Sahni \& Bennet }\end{array}$ & Lanzones & Fruit & Introduced \\
\hline & $\begin{array}{l}\text { Sandoricum koetjape } \\
\text { (Burm.f.) Merr. }\end{array}$ & Santol & Fruit & Native \\
\hline & Swietenia macrophylla King & Mahogany & timber, firewood & Introduced \\
\hline & Melia dubia Cav. & Bangalnga, Baganga & Timber, firewood & Native \\
\hline $\begin{array}{l}\text { Menispermacae } \\
\text { ae }\end{array}$ & Tinospora rumphii Boerl & Panyawan & Baby weaning & Native \\
\hline \multirow[t]{4}{*}{ Moraceae } & Artocarpus heterophyllus Lam. & Nangka & Fruit & Native \\
\hline & Artocarpus odoratissimus Blanco & Marang & Fruit & Native \\
\hline & $\begin{array}{l}\text { Artocarpus altilis } \\
\text { (Parkinson) Fosberg }\end{array}$ & Kolo & Fruit & Native \\
\hline & Ficus benjamina L. & Balite & Ornamental & Native \\
\hline Moringaceae & Moringa oleifera Lam. & $\begin{array}{l}\text { Kalamunggay, } \\
\text { Malunggay }\end{array}$ & $\begin{array}{l}\text { medicinal, } \\
\text { vegetable }\end{array}$ & Introduced \\
\hline Muntingiaceae & Muntingia calabura $\mathrm{L}$. & Mansanitas & Fruit & Introduced \\
\hline Musaceae & Musa paradisiaca $\mathrm{L}$. & Saging & Fruit & Native \\
\hline \multirow[t]{4}{*}{ Myrtaceae } & Psidium guajava L. & Bayabas & Fruit & Introduced \\
\hline & Syzygium aqueum Alston & Tambis & Fruit & Introduced \\
\hline & Syzygium cumini (L.) Skeels. & Lomboy & Fruit & Introduced \\
\hline & $\begin{array}{l}\text { Syzygium mallacense (L.) Merr. \& } \\
\text { L.M.Perry }\end{array}$ & Makopa & Fruit & Introduced \\
\hline Nyctaginaceae & Bougainvillea spectabilis Willd. & Bumbil & Ornamental & Introduced \\
\hline Ochnaceae & Ochna serrulata (Hochst.) Walp. & Mickey Mouse & Ornamental & Introduced \\
\hline Oleaceae & Jasminum sambac (L.) Aiton & Sampaguita & Ornamental & Native \\
\hline \multirow[t]{8}{*}{ Orchidaceae } & Arachnis flos-aeris (L.) Rchb. f. & Spider Orchid & Ornamental & Native \\
\hline & Cattleya Lindl. & Cattleya & Ornamental & Introduced \\
\hline & Dendrobium cf. fairchildiae & Dendrobium & Ornamental & Introduced \\
\hline & Dendrobium anosmum Lindl. & Sanggumay & Ornamental & Native \\
\hline & Oncidium cf. varicosum Lindl. & Dancing Lady & Ornamental & Introduced \\
\hline & Phalaenopsis amabilis (L.) Blume & Butterfly Orchid & Ornamental & Introduced \\
\hline & Spathoglottis plicata Blume & Ground Orchid & Ornamental & Native \\
\hline & Vanda sanderiana Rchb.f. & Vanda & Ornamental & Native \\
\hline \multirow[t]{3}{*}{ Oxalidaceae } & Averrhoa bilimbi L. & Iba & Spice/condiment & Introduced \\
\hline & Averrhoa carambola L. & Balimbing & Fruit & Native \\
\hline & Oxalis regnellii Miq. & Clover & Ornamental & Introduced \\
\hline Pandanaceae & Pandanus amaryllifolius Roxb. & Pandan-humot & Condiment & Introduced \\
\hline Passifloraceae & Passiflora edulis Sims & Valencia & Fruit & Introduced \\
\hline \multirow[t]{2}{*}{ Phyllanthaceae } & Securinega flexuosa Muell.-Arg. & Anislag & Timber & Native \\
\hline & Sauropus androgynus (L.) Merr. & Chinese Malunggay & Vegetable & Introduced \\
\hline Piperaceae & Piper nigrum L. & Paminta & Spice/condiment & Introduced \\
\hline
\end{tabular}


Table 1. Continuation.

\begin{tabular}{|c|c|c|c|c|}
\hline Family & Scientific Name & Local Name & Economical Uses & Origin \\
\hline \multirow[t]{5}{*}{ Poaceae } & Bambusa multiplex (Lour.) Raeusch & Dwarf Bamboo & Ornamental & Introduced \\
\hline & Cymbopogon citratus (DC.) Stapf & Tangad, Tanglad & Spice, Medicinal & \multirow{2}{*}{$\begin{array}{l}\text { Native } \\
\text { Introduced }\end{array}$} \\
\hline & Cynodon dactylon (L.) Pers. & Bermuda & Lawn grass & \\
\hline & Saccharum officinarum L. & Tubo & Sweet sap & Introduced \\
\hline & Saccharum spontaneum Linn. & Bugang-tapol & Medicinal & Exotic \\
\hline Polypodiaceae & Nephrolepis cordifolia & Lukdo-lukdo & Ornamental & \multirow{2}{*}{$\begin{array}{l}\text { Native } \\
\text { Introduced }\end{array}$} \\
\hline Portulacaceae & Portulaca grandiflora Hook. & Vietnam Rose & Ornamental & \\
\hline Rosaceae & Rosa sp.. & Rosas, Rose & Ornamental & Introduced \\
\hline \multirow[t]{4}{*}{ Rubiaceae } & Coffea arabica $\mathrm{L}$. & Kape & Coffee, firewood & Introduced \\
\hline & Ixora chinensis Lam. & Yellow Santan & Ornamental & Introduced \\
\hline & Ixora coccinea $\mathrm{L}$. & Dwarf Santan & Ornamental & Introduced \\
\hline & Mussaenda philippica L. & Dona Aurora & Ornamental & Native \\
\hline \multirow[t]{3}{*}{ Rutaceae } & Citrus grandis Osbeck & Buongon, Pomelo & Vegetable & \multirow{2}{*}{$\begin{array}{l}\text { Native } \\
\text { Introduced }\end{array}$} \\
\hline & Citrus madurensis Lour. & Lemonsito & Condiment & \\
\hline & Citrus L. & Sangkis & Fruit & Introduced \\
\hline Sapindaceae & Nepheleum lappaceum L. & Rambutan & Fruit & \multirow{2}{*}{$\begin{array}{l}\text { Native } \\
\text { Introduced }\end{array}$} \\
\hline Sapotaceae & Chrysophyllum cainito L. & Caimito & Fruit & \\
\hline \multirow[t]{5}{*}{ Solanaceae } & Capsicum annuum L. & Atsal & Spice/condiment & \multirow{2}{*}{$\begin{array}{l}\text { Introduced } \\
\text { Introduced }\end{array}$} \\
\hline & Capsicum frutescens L. (hot chili) & Sili-gagmay & Spice/condiment & \\
\hline & Capsicum sp. (green chili) & Sili-espada & Spice/condiment & \multirow{2}{*}{$\begin{array}{l}\text { Introduced } \\
\text { Introduced }\end{array}$} \\
\hline & Solanum lycopersicon L. & Kamatis & Spice/condiment & \\
\hline & Solanum melongena $\mathrm{L}$. & Talong, Tawong & Vegetable & \multirow{2}{*}{$\begin{array}{l}\text { Introduced } \\
\text { Introduced }\end{array}$} \\
\hline Sterculiaceae & Theobroma cacao L. & Cacao & Fruit, cocoa powder & \\
\hline Tiliaceae & Corchorus olitorius $\mathrm{L}$. & Saluyot & Vegetable & \multirow{2}{*}{$\begin{array}{l}\text { Introduced } \\
\text { Introduced }\end{array}$} \\
\hline Turneraceae & Turnera ulmifolia $\mathrm{L}$. & Turnera & Ornamental & \\
\hline \multirow[t]{4}{*}{ Verbenaceae } & Duranta erecta $\mathrm{L}$. & Duranta & Ornamental & Introduced \\
\hline & Vitex parviflora Juss. & Tugas & Timber & Native \\
\hline & Vitex negundo $\mathrm{L}$. & Lagundi & Medicinal & \multirow{2}{*}{$\begin{array}{l}\text { Native } \\
\text { Introduced }\end{array}$} \\
\hline & Gmelina arborea Roxb. & Yemane & Timber & \\
\hline Xanthorrhoeaceae & Aloe vera (L.) Burm.f. & Sabila & Medicinal & \multirow{2}{*}{$\begin{array}{l}\text { Introduced } \\
\text { Introduced }\end{array}$} \\
\hline \multirow[t]{7}{*}{ Zingiberaceae } & Etlingera elatior (Jack) R.M. Sm. & Torch Ginger & Ornamental & \\
\hline & Zingiber officinale Roscoe & Luy-a & Medicinal, Spice & \multirow{2}{*}{$\begin{array}{l}\text { Introduced } \\
\text { Introduced }\end{array}$} \\
\hline & Curcuma longa $\mathrm{L}$. & Luy-ang Tapol & Medicinal & \\
\hline & Kaempferia galanga $\mathrm{L}$. & Kisol & Medicinal & Introduced \\
\hline & Alpinia elegans (Presl.)Schum & Tagbak & Food, Ornamental & Native \\
\hline & Alpinia purpurata (Vieill.) K. Schum. & Red Ginger & Ornamental & Introduced \\
\hline & Zingiber spectabile Griff. & Yellow Torch Ginger & Ornamental & Native \\
\hline Total no. of fam & Total no. of genera: 146 & Total no. of sp & es: 171 & \\
\hline
\end{tabular}




\section{Vertical stratification}

Forty percent of the home garden plants grown were erect herbs, followed by trees (27\%), shrubs (21\%), herbaceous vines (8\%) and epiphytes (4\%) (Fig 3A). The diversity of species and growth forms shows that the home gardens in the marginal uplands of Inopacan generally exhibited a multi-layered or stratified vertical structure which somehow mimics a tropical forest structure. At the ground layer were erect herbs and creepers and above it was a shrub layer consisting usually of the shrubby ornamentals like the Ti plant (Cordyline fruticosa), San Francisco (Codiaeum variegatum), roses (Rosa spp.), Santan (Ixora spp.); vegetables like okra (Abelmoschus esculentus), eggplant (Solanum melongena) and other crop species. The tree layer consisted mostly of fruit trees such as jackfruit (Artocarpus heterophyllus), mango (Mangifera indica), tambis (Syzigium aqueum), cacao (Theobroma cacao) and some timber and reforestation species like yemane (Gmelina arborea) and narra (Pterocarpus indicus). The climbing species such as string beans (Vigna unguiculata), upo (Lagenaria siceraria), squash (Cucurbita maxima) and ampalaya (Momordica charantia) were usually supported by especially constructed trellises made of bamboo sticks or bamboo branching twigs which the locals called "kagingking". Orchids consisting of vanda (Vanda sanderiana), dancing lady (Oncidium spp.) and several varieties of dendrobium (Dendrobium spp.) and other epiphytic species were either mounted on bamboo posts, on the wiry trunks of the tree fern Cyathea contaminans, or directly attached to the trunks of trees, as in the case of sanggumay (Dendrobium anosmum), a large beautiful native orchid.

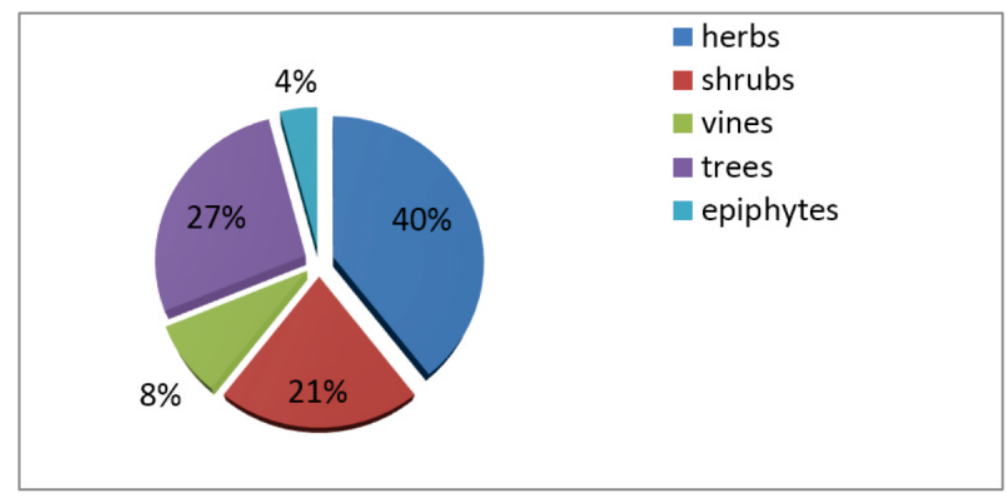

Fig. 3. Distribution of life forms in the home gardens 


\section{Economic Uses}

It is interesting to note that majority of the species grown in the home gardens were ornamentals followed by fruit trees, medicinal plants, vegetables and species used as spices or condiments. The least specious were the root crops. Crops intended for other uses like cacao as source of cocoa powder are represented only by a single species (Fig. 4 \& Table 1 ).

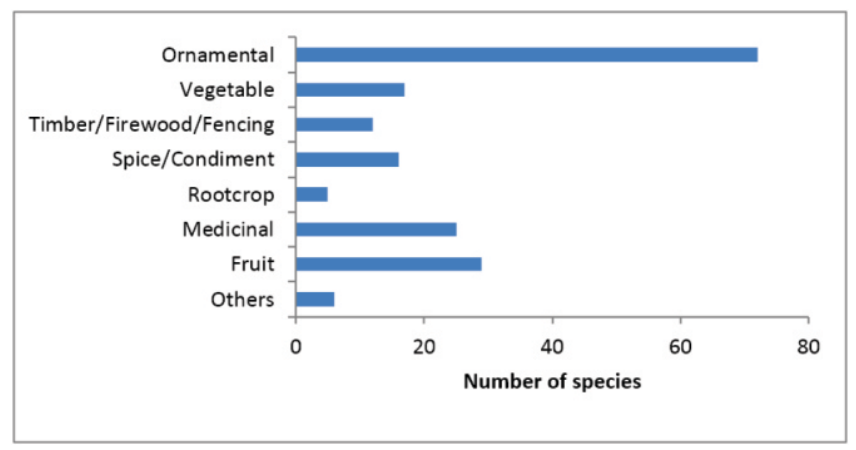

Fig. 4. Economic uses of plants cultivated in home gardens

\section{Medicinal Plants}

Due to distance from hospitals and the high cost of medicine, people in the marginal uplands grew plants in their home gardens for use as herbal medicine which they believed could alleviate or treat common illnesses like cough, fever, toothache, stomachache, loose bowel movement, sore eyes, hyperacidity and muscle pain. Table 2 shows the species of medicinal plants used by the respondents, the ailment/s being treated and the method of preparation.

In $75 \%$ the plant part used in $75 \%$ of the medicinal plant species, the leaves were the plant part used. Other less commonly used parts were the fruits, flower buds, young shoots, roots, bulb, bark, stem, rhizome and endosperm or meat (coconut). The leaves were usually prepared by first heating them over low flame, then crushing using the hands, and applying as poultice over the affected part (57\%). Boiling the leaves in water and drinking the extract or decoction (51\%), or simply putting fresh leaves in a cup of hot water for a few minutes to make an infusion (21\%) were also commonly used methods of preparation. All preparations were done using fresh plant material. In the study of Busman and Sharon (2006), villagers 
of the Lojo Province in Southern Ecuador utilized whole plants (61\%), leaves (13\%), flowers (6\%), and seeds, roots, bark, fruits and latex (3\%), for medicinal purposes.

The medicinal plant species documented were used to treat one to as many as seven ailments. Yerba Buena (Mentha cordifolia) is top of the list, reported by the respondents to cure ear infection, flatulence, muscle pain, stomachache, cough, hyperacidity and as an effective dewormer. Mayana (Plechranthus blumei) was reported to be a cure for five diseases, namely; cough, fever, red eyes, itchy eyes and for healing wounds. Guyabano (Annona muricata) was also used to treat simple ailments or discomforts like hyperacidity, loose bowel movement and flatulence, to more serious ones like cancer. The local folks mentioned that they also use guyabano to boost the immune system. The common Madre de Cacao or Kakawate (Glericidia sepium) was considered effective for wounds and fractures in humans as well as flea infestation, warts and skin diseases of goats, chicken and pigs, respectively.

\section{Establishment and management of homegardens}

\section{Sources of planting materials}

The planting materials used in home gardening were obtained from different sources (Fig. 5). More than half (58\%) of the respondents got their planting materials for free, shared by relatives and friends. About a quarter (23\%) simply gathered planting materials from their surroundings or collected from the wild. Only 14\% purchased their planting materials and $5 \%$ obtained them in exchange for another plant found in their garden.

It seems evident that majority of those having home gardens did not prioritize purchase of planting materials in their family budget, especially for non-food plants like ornamentals. This is understandable as families living in the uplands mostly live below the poverty line so their priority must be their daily subsistence.

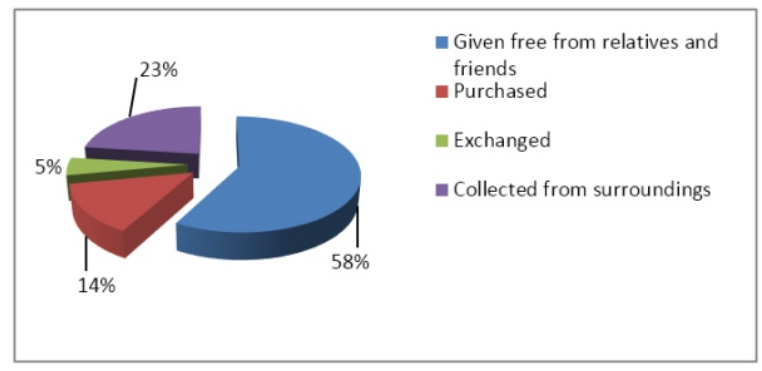

Fig. 5. Sources of planting materials for the home gardens 
Table 2. Species of medicinal plants grown in the marginal uplands home gardens

\begin{tabular}{|c|c|c|c|c|}
\hline Scientific name & Local name & Ailment (s) Treated & $\begin{array}{l}\text { Plant Part } \\
\text { Used }\end{array}$ & Mode of Preparation \\
\hline \multirow[t]{2}{*}{ Artemisia vulgaris L. } & Hilbas & $\begin{array}{l}\text { Cough, flatulence, muscle } \\
\text { pain }\end{array}$ & Leaves & $\begin{array}{l}\text { Slightly heat leaves over low fire. } \\
\text { Crush leaves and rub on affected } \\
\text { part. }\end{array}$ \\
\hline & & Painful urination & Whole plant & $\begin{array}{l}\text { Boil leaves in water and drink } \\
\text { the decoction. }\end{array}$ \\
\hline \multirow[t]{5}{*}{$\begin{array}{l}\text { Plechranthus blumei } \\
\text { Benth. }\end{array}$} & Mayana/Manjana & Cough & Leaves & $\begin{array}{l}\text { Boil leaves in water and drink } \\
\text { the decoction. }\end{array}$ \\
\hline & & Fever & Leaves & $\begin{array}{l}\text { Put leaves in a cup of hot water. } \\
\text { Let it cool and drink the infusion. } \\
\text { Alternatively, heat leaves over } \\
\text { low fire, crush and apply as } \\
\text { poultice on the forehead. }\end{array}$ \\
\hline & & Wound & Leaves & $\begin{array}{l}\text { Slightly heat leaves over low } \\
\text { fire. Crush and rub on the } \\
\text { affected part. }\end{array}$ \\
\hline & & Red eyes & Leaves & $\begin{array}{l}\text { Slightly heat leaves and place } \\
\text { over infected eyes. }\end{array}$ \\
\hline & & Itchy eyes & Leaves & $\begin{array}{l}\text { Crush leaves and squeeze the } \\
\text { extract. Put a drop of extract to } \\
\text { itching eye. }\end{array}$ \\
\hline $\begin{array}{l}\text { Tradescantia spathacea } \\
\text { Sw. }\end{array}$ & Bangka-bangkaan & Fever from measles & Leaves & $\begin{array}{l}\text { Put leaves in a cup of hot water. } \\
\text { Let it cool and drink the infusion. }\end{array}$ \\
\hline Vitex negundo L. & Lagundi & Cough & Leaves & $\begin{array}{l}\text { Boil leaves and drink the } \\
\text { decoction or put a few leaves in a } \\
\text { cup of hot water and drink the } \\
\text { infusion. }\end{array}$ \\
\hline \multirow[t]{2}{*}{ Persea americana Mill. } & Avocado & Loose bowel movement & Leaves & $\begin{array}{l}\text { Boil leaves and drink the } \\
\text { decoction. }\end{array}$ \\
\hline & & Hyperacidity & Leaves & $\begin{array}{l}\text { Crush the leaves and rub on the } \\
\text { stomach area. }\end{array}$ \\
\hline
\end{tabular}


Table 2 Continuation.

\begin{tabular}{|c|c|c|c|c|}
\hline Scientific Name & Local Name & Ailment (s)/ Treatment & $\begin{array}{l}\text { Plant Part } \\
\text { Used }\end{array}$ & Mode of Preparation \\
\hline Citrus madurensis Lour. & Lemonsito & Cough & Fruit & $\begin{array}{l}\text { Put extracted juice in a cup of } \\
\text { water, add a tablespoon of honey } \\
\text { and drink it. }\end{array}$ \\
\hline \multirow[t]{2}{*}{ Annona muricata $\mathrm{L}$. } & Abana/Guyabano & $\begin{array}{l}\text { Hyperacidity, Loose } \\
\text { bowel movement, } \\
\text { Flatulence }\end{array}$ & Leaves & $\begin{array}{l}\text { Boil the leaves and drink the } \\
\text { decoction. }\end{array}$ \\
\hline & & $\begin{array}{l}\text { Cancer, Boost immune } \\
\text { system, }\end{array}$ & Fruit & $\begin{array}{l}\text { Boil the leaves and drink the } \\
\text { decoction. }\end{array}$ \\
\hline Hibiscus rosa-sinensis L. & Antuwanga & $\begin{array}{l}\text { Boils, wound, skin rashes, } \\
\text { sore eyes }\end{array}$ & Flower buds & $\begin{array}{l}\text { Mash the flower buds and apply } \\
\text { as poultice on the infected area. }\end{array}$ \\
\hline Musa sp. & Saging & Boils & Pseudostem & $\begin{array}{l}\text { Finely scrape the outer part of } \\
\text { the pseudostem and apply as } \\
\text { poultice on the affected part. }\end{array}$ \\
\hline Mikania cordata & Vietnam, Asyang & Wound & Leaves & $\begin{array}{l}\text { Crush the leaves and apply as } \\
\text { poultice on the wounded area. }\end{array}$ \\
\hline \multirow[t]{5}{*}{$\begin{array}{l}\text { Gliricidia sepium (Jacq.) } \\
\text { Kunth ex Walp. }\end{array}$} & Madre cacao & Wound & Leaves & $\begin{array}{l}\text { Crush the leaves and apply as } \\
\text { poultice on the wounded area. }\end{array}$ \\
\hline & & Fractures & Leaves & $\begin{array}{l}\text { Crush the leaves and apply as } \\
\text { poultice on the affected area. }\end{array}$ \\
\hline & & Fleas infestation in goats & Leaves & $\begin{array}{l}\text { Crush or pound the leaves and } \\
\text { rub on body of the goat. }\end{array}$ \\
\hline & & Warts of chicken & Leaves & $\begin{array}{l}\text { Crush the leaves and put the } \\
\text { extract on the warts. }\end{array}$ \\
\hline & & Skin disease of pigs & Leaves & $\begin{array}{l}\text { Crush the leaves and rub on the } \\
\text { affected part. }\end{array}$ \\
\hline \multirow[t]{2}{*}{ Psidium guajava L. } & Guava & Wound & Leaves & $\begin{array}{l}\text { Crush the leaves and put on the } \\
\text { wounded part. }\end{array}$ \\
\hline & & $\begin{array}{l}\text { Loose bowel movement, } \\
\text { indigestion }\end{array}$ & Leaves & $\begin{array}{l}\text { Boil the leaves and drink the } \\
\text { decoction. }\end{array}$ \\
\hline
\end{tabular}


Table 2 Continuation.

\begin{tabular}{|c|c|c|c|c|}
\hline Scientific Name & Local Name & Ailment (s)/ Treatment & $\begin{array}{l}\text { Plant Part } \\
\text { Used }\end{array}$ & Mode of Preparation \\
\hline Chrysophyllum cainito L. & Caimito & $\begin{array}{l}\text { Loose bowel, Flatulence, } \\
\text { Hyperacidity }\end{array}$ & Leaves & $\begin{array}{l}\text { Boil the leaves and drink the } \\
\text { decoction. }\end{array}$ \\
\hline \multirow[t]{2}{*}{$\begin{array}{l}\text { Saccharum spontaneum } \\
\text { L. }\end{array}$} & \multirow[t]{2}{*}{ Bugang-tapol } & Vomiting blood & $\begin{array}{l}\text { Young fleshy } \\
\text { shoot }\end{array}$ & $\begin{array}{l}\text { Chew the young shoot and swallow } \\
\text { the juice. }\end{array}$ \\
\hline & & Diabetes, high blood & young shoot & $\begin{array}{l}\text { Cut the stem/culm into small pieces, } \\
\text { boil in water and drink the decoction. }\end{array}$ \\
\hline \multirow[t]{2}{*}{$\begin{array}{l}\text { Plechranthus aromaticus } \\
\text { Benth. }\end{array}$} & \multirow[t]{2}{*}{ Karabo } & Cough & Leaves & $\begin{array}{l}\text { Boil the leaves and drink the } \\
\text { decoction or crush slightly heated } \\
\text { leaves and rub on the chest and back. }\end{array}$ \\
\hline & & Repelling mosquitoes & Leaves & $\begin{array}{l}\text { Put freshly crushed leaves in a plate } \\
\text { and let the smell diffuse out to repel } \\
\text { mosquitoes. }\end{array}$ \\
\hline Moringa oleifera Lam. & Kalamunggay & Wound & Leaves & $\begin{array}{l}\text { Crush the leaves and apply as poultice } \\
\text { on the wounded part. }\end{array}$ \\
\hline $\begin{array}{l}\text { Sanseveira trifasciata } \\
\text { Hort. ex Prain }\end{array}$ & Espada-espada & Wound & Leaves & $\begin{array}{l}\text { Squeeze out the juice and apply the } \\
\text { extract on the wounded part. }\end{array}$ \\
\hline Cocos nucifera $\mathrm{L}$. & Lubi & Wound & $\begin{array}{l}\text { Solid } \\
\text { endosperm } \\
\text { (meat) }\end{array}$ & $\begin{array}{l}\text { Lightly scrape the soft surface of the } \\
\text { coconut meat and apply as poultice on } \\
\text { the wounded part. }\end{array}$ \\
\hline Kaempferia galanga $\mathrm{L}$. & Kisol & Dry cough & Bulb & $\begin{array}{l}\text { Tie the bulb around the neck like a } \\
\text { necklace. }\end{array}$ \\
\hline Allium sp & Sibojing & Neck pain in children & Leaves & $\begin{array}{l}\text { Slightly heat the leaves and extract the } \\
\text { juice. Massage the extract on the neck. }\end{array}$ \\
\hline Mentha arvensis $\mathrm{L}$. & Menthol plant & Cough & Leaves & Chew the leaves to soothe the throat. \\
\hline Jatropha gossypifolia $\mathrm{L}$. & Tuba-tuba tapol & Flatulence & Leaves & $\begin{array}{l}\text { Slightly heat the leaves and extract the } \\
\text { juice. Massage the juice on the neck. }\end{array}$ \\
\hline
\end{tabular}


Table 2 Continuation.

\begin{tabular}{|c|c|c|c|c|}
\hline Scientific Name & Local Name & Ailment(s)/ Treatment & $\begin{array}{l}\text { Plant Part } \\
\text { Used }\end{array}$ & Mode of Preparation \\
\hline Jatropha curcas & Tuba-tuba & Stomachache & Leaves & $\begin{array}{l}\text { Slightly heat the leaves over low } \\
\text { flame and spread on the stomach } \\
\text { area. }\end{array}$ \\
\hline \multirow[t]{2}{*}{$\begin{array}{l}\text { Hyptis suaveolens (L.) } \\
\text { Poit }\end{array}$} & \multirow[t]{2}{*}{ Albahaka } & $\begin{array}{l}\text { Stomach pain, Loose } \\
\text { bowel movement }\end{array}$ & Roots & $\begin{array}{l}\text { Boil the roots and drink the } \\
\text { decoction. }\end{array}$ \\
\hline & & Wound & Leaves & $\begin{array}{l}\text { Crush the leaves and apply as } \\
\text { poultice on the wounded area. }\end{array}$ \\
\hline $\begin{array}{l}\text { Kalanchoe pinnata } \\
\text { (Lam.) Pers. }\end{array}$ & Hanlilika/Katakataka & Boil, Toothache & Leaves & $\begin{array}{l}\text { Crush the leaves and put it over } \\
\text { the boil or the aching tooth. }\end{array}$ \\
\hline Adiantum sp. & Lamon-lamon & Infected wound & Leaves & $\begin{array}{l}\text { Crush leaves and apply as } \\
\text { poultice on the infected wound. }\end{array}$ \\
\hline $\begin{array}{l}\text { Lagerstroemia speciosa } \\
\text { (L.)Pers. }\end{array}$ & Banaba & Kidney infection & Leaves & $\begin{array}{l}\text { Boil } 7 \text { mature leaves in } 7 \text { glasses } \\
\text { of water and drink the decoction. }\end{array}$ \\
\hline $\begin{array}{l}\text { Syzygium cuminii (L.) } \\
\text { Skeels }\end{array}$ & Lomboy & Toothache & Bark & $\begin{array}{l}\text { Boil the bark for } 15 \mathrm{~min} \text { and } \\
\text { drink the decoction. }\end{array}$ \\
\hline $\begin{array}{l}\text { Cymbopogon citratus } \\
\text { (DC) Stapf. }\end{array}$ & Tangad & High blood & Leaves & $\begin{array}{l}\text { Boil the leaves and drink the } \\
\text { decoction. }\end{array}$ \\
\hline \multirow[t]{4}{*}{ Mentha cordifolia Opiz } & \multirow[t]{4}{*}{ Yerba Buena } & Ear infection & Leaves & $\begin{array}{l}\text { Slightly heat the leaves and } \\
\text { insert into the outer part of the } \\
\text { ear. }\end{array}$ \\
\hline & & $\begin{array}{l}\text { Flatulence, body pain, } \\
\text { stomachache }\end{array}$ & Leaves & $\begin{array}{l}\text { Crush the leaves and rub on } \\
\text { affected part. }\end{array}$ \\
\hline & & Cough, Hyperacidity & Leaves & $\begin{array}{l}\text { Put the leaves in a cup, pour hot } \\
\text { water. Let it cool and drink the } \\
\text { water/infusion. }\end{array}$ \\
\hline & & Stomach worms & Leaves & $\begin{array}{l}\text { Put the leaves in a cup, pour hot } \\
\text { water. Let it cool and drink the } \\
\text { water/infusion. }\end{array}$ \\
\hline $\begin{array}{l}\text { Colocasia esculenta (L.) } \\
\text { Schott }\end{array}$ & Gabi & Wound & Petiole & $\begin{array}{l}\text { Scrape the surface of the petiole } \\
\text { and apply as poultice on the } \\
\text { wounded part. }\end{array}$ \\
\hline
\end{tabular}


Table 2 Continuation.

\begin{tabular}{|c|c|c|c|c|}
\hline Scientific Name & Local Name & Ailment (s)/ Treatment & $\begin{array}{l}\text { Plant Part } \\
\text { Used }\end{array}$ & Mode of Preparation \\
\hline \multirow[t]{3}{*}{ Tinospora rumphii Boerl. } & Panyawan & Anemia & Stem & $\begin{array}{l}\text { Cut the stem into pieces and boil } \\
\text { in water. Mix the extract in the } \\
\text { water for bathing. }\end{array}$ \\
\hline & & Stomachache & Stem & $\begin{array}{l}\text { Cut the stem into pieces and boil } \\
\text { in water. Drink the water. }\end{array}$ \\
\hline & & Weaning of babies & Stem & $\begin{array}{l}\text { Rub the stem sap on the } \\
\text { mother's nipple. }\end{array}$ \\
\hline \multirow[t]{3}{*}{$\begin{array}{l}\text { Alpinia purpurata } \\
\text { (Vieill.) K. Schum. }\end{array}$} & Luy-a tapol & Body pain & Rhizome & $\begin{array}{l}\text { Pound the rhizome and rub on } \\
\text { affected part. }\end{array}$ \\
\hline & & Hoarse throat & Rhizome & $\begin{array}{l}\text { Get a slice of the rhizome and use } \\
\text { it like candy. }\end{array}$ \\
\hline & & Cough & Rhizome & $\begin{array}{l}\text { Pound the rhizome and rub on } \\
\text { the chest and back. }\end{array}$ \\
\hline \multirow[t]{2}{*}{$\begin{array}{l}\text { Orthosiphon aristatus } \\
\text { (Blume) Miq }\end{array}$} & Balbaspusa & Kidney infection & Leaves & $\begin{array}{l}\text { Boil the leaves and drink the } \\
\text { decoction. }\end{array}$ \\
\hline & & Cough, fever & Leaves & $\begin{array}{l}\text { Boil the leaves and drink the } \\
\text { decoction. }\end{array}$ \\
\hline Bixa orellana $\mathrm{L}$. & Atsuete & Flatulence, fracture & Leaves & $\begin{array}{l}\text { Spread fresh leaves on the } \\
\text { affected part. }\end{array}$ \\
\hline Centella asiatica $\mathrm{L}$. & Jahong-jahong & Fever & whole plant & $\begin{array}{l}\text { Put whole plant in a glass of } \\
\text { warm water and drink the water. }\end{array}$ \\
\hline Euphorbia neriifolia L. & Suro-suro & $\begin{array}{l}\text { Pierced by a nail or other } \\
\text { sharp objects }\end{array}$ & $\begin{array}{l}\text { stem and } \\
\text { leaves }\end{array}$ & $\begin{array}{l}\text { Put a drop of plant sap on the } \\
\text { affected part for } 4 \text { days }\end{array}$ \\
\hline \multirow[t]{2}{*}{$\begin{array}{l}\text { Blumea balsamifera (L.) } \\
\text { DC }\end{array}$} & Gabon & Flatulence & Leaves & $\begin{array}{l}\text { Boil the leaves and drink the } \\
\text { decoction. }\end{array}$ \\
\hline & & Kidney infection & Leaves & $\begin{array}{l}\text { Put whole plant in a glass of } \\
\text { warm water and drink the water. }\end{array}$ \\
\hline Urena lobata & Daupang & Wound in animals & Leaves & $\begin{array}{l}\text { Pound the leaves and extract the } \\
\text { juice. Use the extract to clean the } \\
\text { wound. }\end{array}$ \\
\hline
\end{tabular}




\section{Mode of cultivation}

The locals used different methods of putting up their home gardens (Fig. 6A). About half of them (46\%) did plotless planting or direct planting in the soil. Species like malunggay (Moringa oleifera), ornamental plants like santan (Ixora spp.) periwinkle or kumintang (Catharanthus roseus), bombil (Bougainvillea spectabilis) and golden duranta (Duranta erecta) and other tree species were usually planted using this method. About the same number of respondents used containers such as clay or plastic pots, polyethylene or ordinary recycled plastic bags while some recycled empty milk cans and similar containers. Vegetables like spring onions (Allium fistolusum) and ornamental plants like roses, bromeliads and cactuses were usually raised using this method. Only $7 \%$ of the respondents grew their plants in raised plots and was usually done for vegetables, especially if a portion of the harvested products was sold in the local market. Pechay (Brassica rapa), tomatoes (Solanum lycopersicum), bell pepper (Capsicum annuum) and eggplant (Solanum melongena) were the common plants grown in raised plots.

More than half $(62 \%)$ of the respondents grew plants in home gardens for home consumption. About a third (35\%) did it as source of additional income for the family while only $5 \%$ did it to beautify their surroundings (Fig. 6B). Cultivating the home gardens was mostly a family affair with members of the family providing labor (96\%) and only a very small percentage (4\%) hired laborers (Fig. 6C). The time the family members spent together in the garden helped build stronger family ties. Almost all of them (89\%) did not apply fertilizer (Fig. 6D), and those who did used organic fertilizer (87\%) derived from composted plant debris or animal manure such as chicken dung or goat manure (Fig. 6E). Since home gardens usually involved only small patches of land mostly used to provide additional food for daily subsistence, the family would use every opportunity to use free labor and production materials that are locally available and cost-free. 

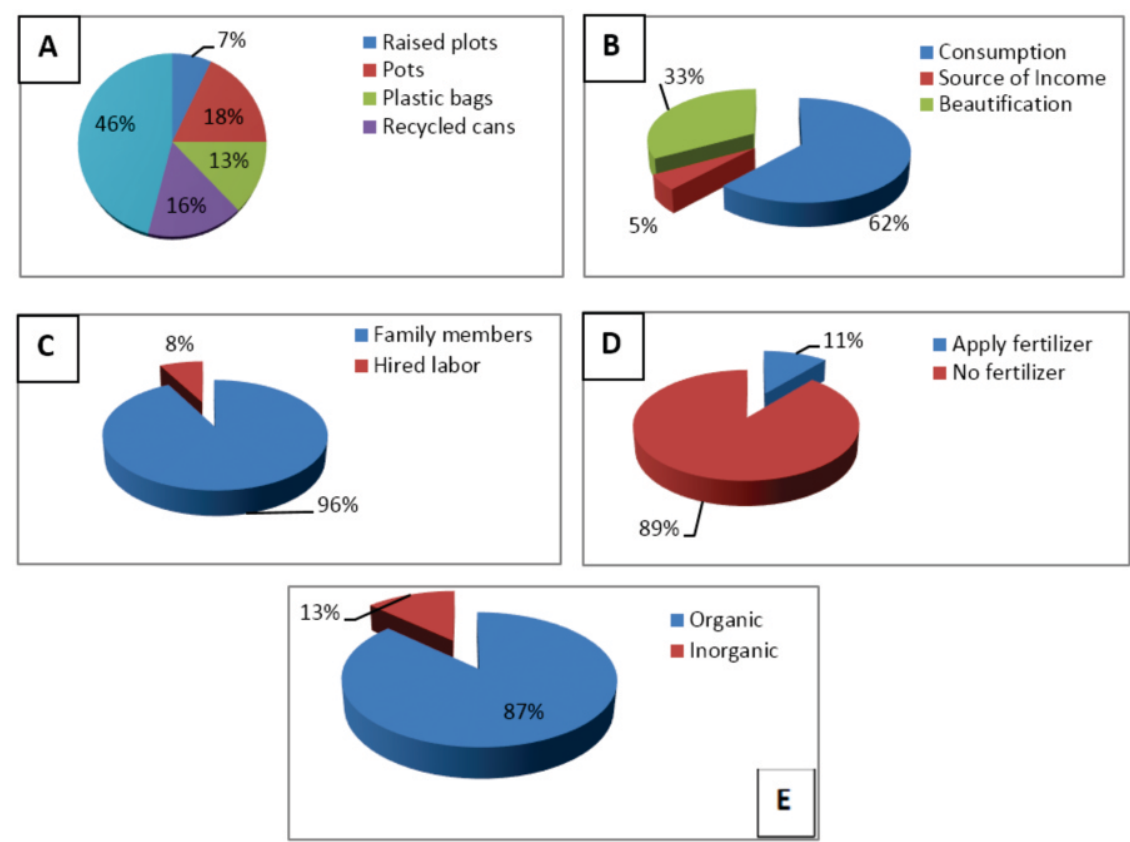

Fig. 6. Method of planting (A), reason for cultivating (B), source of labor (C), fertilizer application (D) and type of fertilizer used (E)

\section{CONCLUSION AND RECOMMENDATIONS}

Cultivating a home garden has indeed become an important subsistence and coping strategy of upland villages especially in the highly vulnerable marginal uplands. Based on this study, the home gardens of Inopacan, Leyte, contain a great diversity of species and varieties which the locals collect through donations, exchange and collection from their surroundings. The more personalized management of the gardens by family members further make home gardening an effective strategy for biodiversity conservation. Thus, the importance of home gardening in the conservation of biodiversity, especially for economically important species, should be given preferential attention in the present global efforts to halt or at least reduce biodiversity loss. However, there is need to effectively educate local villages on the importance of native species because of the continued patronage of exotic or introduced species, which can have drastic effects to the environment. 


\section{ACKNOWLEDGMENT}

The authors wish to thank the Commission on Higher EducationPhilippine Higher Education Research Network (CHED-PHERNet) for the financial support of the research.

\section{REFERENCES}

ASIO, V.B., A. B. BOLLEDO, S.B. LINA, D.S. MARANGUIT, R.J.T. DOGUILES, and K. L.N. DEMAIN. 2014. Characteristics of soils in the marginal uplands of Inopacan, Leyte. Annals of Trop. Res. 36(3):1-14.

BUCHMANN, C. 2009. Cuban home gardens and their role in social-ecological resilience. Human Ecology 37:705-721

COOMES, O.T. and N. BAN. 2004. Cultivated plant species diversity in home gardens of an Amazonian peasant village in northeastern Peru. Economic Botany 58(3): 420-434.

EYZAGUIRRE P. and J. WATSON. 2001 Home gardens and agrobiodiversity: an overview across regions. In: Watson, J.W. and P.B. Eyzaguirre .(eds.) Proceedings of the second international home garden workshop. Bioversity International, Rome, Italy, pp 10-13

GALLUZZI, G., P. EYZAGUIRRE and V. NEGRI. 2010. Home gardens: neglected hotspots of agro-biodiversity and cultural diversity. Bioversity Conservation 19:3635-3654.

GUARINO L. and M. HOOGENDIJK. 2004. Microenvironments. In: Eyzaguirre P. and O. Linares. (eds.) Home gardens and agrobiodiversity. Smithsonian Books, Washington, pp 31-40

HODGKIN T. 2001. Home gardens and the maintenance of genetic diversity. In: Watson, J.W. and P.B. Eyzaguirre .(eds.) Proceedings of the second international home garden workshop. Bioversity International, Rome, Italy, pp 14-18 
Agrobiodiversity of home gardens in marginal upland Villages of Inopacan

KULPA, W. and P. HANELT.1981. Activities regarding collection and evaluation of Polish landraces. Kulturpflanze 29:81-90

LEIVA J.M., C. AZURDIA and W. OVANDO. 2001. Contributions of home gardens to in situ conservation in traditional farming systems-Guatemalan component. In: Watson, J.W. and P.B. Eyzaguirre .(eds.) Proceedings of the second international home garden workshop. workshop. Biodiversity International, Rome, Italy, pp 56-72

PELSER, P.B., J.F. BARCELONA, \& D.L. NICKRENT (eds.). 2011- onwards. Co's Digital Flora of the Philippines. www.philippineplants.org.

STEENIS, C.G.G.J. van (ed.). 1950- onwards. Flora Malesiana. Series I, Spermatophyta. Biodiversity Heritage Library. http://www.biodiversitylibrary.org.

SUNWAR, S., C.G. THORNSTROM, A. SUBEDI and M. BYSTROM. 2006. Home gardens in western Nepal: Opportunities and challenges for on-farm management of agrobiodiversity. Biodiversity and Conservation 15:4211-4238.

TRINH, L.N., J.W. WATSON, N.N. HUE, N.N. DE, N.V. MINH, P. CHU, B.R. STHAPIT , and P.B. EYZAGUIRRE. 2003. Agrobiodiversity conservation and development in Vietnamese home gardens. Agriculture Ecosystems and Environment 97:317-344. 